\title{
Vulnerabilidade ao estresse no trabalho e percepção de suporte familiar em porteiros: um estudo correlacional
}

\author{
Cristiane Oliveira Alves Telles Nunes - Universidade Federal de São Carlos, São Carlos, Brasil \\ Sandra Leal Calais' - Universidade Estadual Paulista Júlio de Mesquita Filho, Bauru, Brasil
}

\begin{abstract}
Resumo
Este estudo objetivou avaliar e correlacionar os níveis de vulnerabilidade ao estresse no trabalho e percepção de suporte familiar em trabalhadores porteiros. Participaram 100 porteiros de uma empresa prestadora de serviços de médio porte. Utilizou-se o questionário de caracterização, a Escala de Vulnerabilidade ao Estresse no Trabalho (EVENT) e o Inventário de Percepção de Suporte Familiar (IPSF). A idade média dos participantes foi de 37,8 anos; trabalhavam na empresa, em torno de quatro anos e como porteiros, em média, há cinco anos. Os resultados mostraram uma pontuação média na medida de estresse geral da EVENT, sugerindo indicação de poucos estressores neste grupo de porteiros. O IPSF também apresentou pontuação média, indicando que grande parte dos participantes possui percepção de bom suporte familiar. As análises mostraram correlações negativas fracas, porém estatisticamente significantes, entre a dimensão Adaptação Familiar do IPSF e as dimensões Clima e Funcionamento Organizacional, Pressão no Trabalho e Total da EVENT.

Palavras-chave: Porteiros, Vulnerabilidade ao estresse, Trabalho, Percepção de suporte familiar.
\end{abstract}

Vulnerability to the stress at work and perception of family support in doormen: a correlational study

\begin{abstract}
This study had the objective of evaluating and correlating the levels of work stress vulnerability and family support perception in doormen workers. 100 doormen of a medium sized, service provider company took part. A characterization questionnaire, the Scale of Vulnerability to the Stress at Work (EVENT), and the Inventory of Perception of Family Support (IPSF) were used. The age average of the participants was 37,8 years old, who worked in the company around four years, and as doormen, an average of five years. The results showed an average score that was obtained in measuring general stress of EVENT, suggesting that few stress factors were indicated on this doormen group. The IPSF also presented an average score, indicating that most of the participants have the perception of good family support. The analysis showed negative correlations between work stress vulnerability and family support, but statistically significant.

Keywords: Doormen, Stress vulnerability, Work, Family support perception.
\end{abstract}

Alguns estudos têm sido realizados com o objetivo de investigar as variáveis que influenciam o estresse ocupacional, fenômeno constantemente associado à saúde do trabalhador e ao desempenho organizacional. $O$ estresse ocupacional pode ser definido como um processo em que a pessoa percebe demandas do trabalho como estressoras, as quais, ao exceder sua habilidade de enfrentamento, provocam reações negativas no indivíduo (Paschoal \& Tamayo, 2004, 2005).

Outras pesquisas sobre estresse incluem o estudo dos seus efeitos negativos no que se refere à profissão (Lipp \& Tanganelli, 2002). Diferentes profissões foram estudadas e, no Brasil, encontram-se pesquisas sobre o estresse ocupacional com bombeiros (Calais, 2004), psicólogos (Covolan, 2001), atletas (Maciel, 2001), juízes e servidores públicos (Oliveira, 2004), militares (Romano, 2001), etc.

${ }^{1}$ Endereço para correspondência:

Universidade Estadual Paulista - UNESP

Departamento de Psicologia

Av. Eng. Luiz Edmundo Carrijo Coube, 14-01 - 17033-360 Bauru-SP, Brasil.

E-mail: scalais@fc.unesp.br
Os estudos sobre estresse em diferentes profissões consideram, evidentemente, as especificidades potencialmente estressantes das funções que exercem essas pessoas na sua ocupação. Nos estudos da literatura é possível perceber que, em geral, essas profissões eventualmente possuem a exigência de uma formação acadêmica prolongada, isto é, investimentos altos para uma formação adequada, além de serem profissionais de uma classe social e econômica mais favorecida: juízes, funcionários públicos, professores, psicólogos e médicos. Neste sentido, supõe-se que resultados diferentes podem ser encontrados em uma população que exerce a profissão de porteiro, já que esta, apesar de possuir possíveis estressores inerentes à função, não exige formação acadêmica e em geral é exercida por pessoas da classe trabalhadora menos favorecida.

O ritmo acelerado da sociedade atual faz com que a maioria das pessoas desenvolva estresse em algum momento da vida (Ferachin, Ranzi, Garib \& Lopes, 2004). Lipp (2000) define o estresse como uma reação complexa e global do organismo que envolve componentes físicos, psicológicos e hormonais, desenvolvendo-se em etapas ou fases. Portanto, estresse não é uma doença, mas um alerta do corpo para o período entre a saúde e a doença (Lipp, 1999). 
Considera-se, também, que o estresse envolve uma relação singular entre o indivíduo e seu ambiente, sendo percebido pela pessoa como uma ameaça ou ainda como algo que exige dela mais que suas próprias capacidades ou recursos, colocando em risco seu bemestar (Limongi-França \& Rodrigues, 2005).

Apesar de vários autores apresentarem considerações sobre o estresse com um número significativo de publicações sobre o tema, ainda existem dúvidas, mitos e dificuldades conceituais acerca do construto (Zanelato, 2008). De acordo com Mota, Franco e Motta (1999), as dificuldades de conceituação do estresse relacionam-se à existência de muitos modelos teóricos, visto que alguns o caracterizam em termos de respostas, outros o descrevem como um conjunto de estímulos e ainda há aqueles que sugerem que o estresse pode ser desencadeado pela falta de fortalecimento do indivíduo, dos efeitos da falta de suporte para a adaptação e de ameaças ambientais, focando a relação entre o ambiente e o homem. Lipp (1999) também relata que, ora o fenômeno estresse é descrito como um estímulo que gera uma quebra na homeostase, ora é descrito como uma resposta comportamental criada por tal desequilíbrio. Assim, o estresse pode ser caracterizado como uma condição, causa ou estímulo desencadeante de uma reação do organismo.

Alguns pesquisadores estudaram o estresse por meio da sua vulnerabilidade no ambiente de trabalho. Bachion, Abreu, Godoy e Costa (2005) realizaram um estudo para estimar a vulnerabilidade ao estresse em professores universitários, relacionando-a com algumas características sociodemográficas dos docentes. Participaram 71 professores e a maioria $(76,1 \%)$ apresentou baixa vulnerabilidade ao estresse, $21,1 \%$ revelaram moderada vulnerabilidade e $2,8 \%$ alta vulnerabilidade. Constatou-se tendência à maior vulnerabilidade em mulheres com até 40 anos, sem companheiro, com três ou quatro áreas de atuação e que possuem apenas especialização.

Miguel e Noronha (2007) realizaram um estudo com o objetivo de buscar evidências de validade para a Escala de Vulnerabilidade ao Estresse do Trabalho - EVENT (Sisto, Baptista, Noronha \& Santos, 2007) por intermédio de parâmetros psicométricos. Participaram da pesquisa 116 pessoas, sendo 85 do sexo feminino, com idade variando de 19 a 67 anos. Os resultados mostraram que a EVENT não apresentou correlação significativa com idade e escolaridade. Encontrou-se diferença significativa de média entre sexos apenas para o segundo fator da EVENT, Pressão no trabalho. Os homens obtiveram média de 22,26 enquanto as mulheres de 24,79, sugerindo que elas tendem a perceber, mais do que os homens, a pressão no trabalho.

Portanto, faz-se necessário realizar estudos que correlacionem a vulnerabilidade ao estresse no trabalho com outras variáveis identificando os fatores que podem interferir positiva e negativamente na vida pessoal e profissional do indivíduo. Um desses fatores pode ser a família que a pessoa possui.

A família é considerada um sistema muito importante na rede de apoio social do indivíduo, seja pelo seu papel de provedora e cuidadora, seja como agente socializador. Destaca-se como a responsável por oferecer as primeiras relações estáveis e de confiança, o que se reflete nos sistemas de crenças e valores do indivíduo (Poletto \& Koller, 2002).

A função e a estrutura da família vêm sofrendo grandes mudanças nas últimas décadas, acompanhando as tendências de desenvolvimento nos âmbitos econômico, político, cultural e tecnológico. Contudo, ainda que alguns fenômenos venham gerando questionamentos sobre a centralidade e o futuro da família nas sociedades contemporâneas, suas responsabilidades e funções sociais não parecem ter perdido a importância (Baptista, Baptista \& Dias, 2001; Carvalho \& Almeida, 2003).

Segundo Aquino (2007), para compreender o termo família, faz-se necessário diferenciar os conceitos de estrutura e suporte familiar. Compreende-se por estrutura as características físicas da família, dentre elas, o número de pessoas que a compõe e como estão dispostas, se é nuclear ou foi dividida. O suporte familiar, por sua vez, relaciona-se com as variáveis psicológicas presentes nas relações familiares como expressão de carinho, atenção, comunicação, proximidade afetiva, permissão de autonomia, liberdade, superproteção, independência, diálogo, entre outros (Baptista \& Oliveira, 2004).

Mota, Franco e Motta (1999) enfatizaram que receber suporte adequado pode contribuir para capacitar a família a funcionar com maiores recursos e serem mais versáteis. O suporte familiar agiria como protetor para eventos vitais estressantes à medida que estaria presente e principalmente percebido como disponível.

Baptista (2005) relata que o suporte familiar é fundamental nos estudos de resiliência psicológica por ser considerado um dos mais importantes amortecedores do efeito de vários estressores na vida das pessoas. O suporte social, como uma condição ampla, e o suporte familiar, como uma condição específica, são ambos processos importantes para a manutenção da saúde mental e enfrentamento de situações estressantes (Baptista, Baptista \& Torres, 2006). 
Considerando a importância da família em vários âmbitos da vida do indivíduo, Baptista (2005; 2010) desenvolveu o Inventário de Percepção de Suporte Familiar (IPSF) que avalia a forma como a pessoa percebe sua relação com a família. Nem sempre o suporte real existente na família é, de fato, percebido pelos membros familiares, por isso os estudos nessa área investigam a percepção do suporte, uma vez que é esta percepção que atua nos indivíduos. Assim, muitos instrumentos disponíveis de avaliação mensuram a percepção do suporte e não o suporte efetivamente oferecido pela família.

Nos últimos anos o IPSF tem sido utilizado em diversas pesquisas e destaca-se o estudo de Aquino (2007) que objetivou buscar evidências de validade entre o Inventário de Percepção de Suporte Familiar (IPSF) e a Escala de Vulnerabilidade ao Estresse no Trabalho (EVENT). O estudo foi realizado com universitários de uma instituição particular da cidade de São Paulo e os resultados, no geral, mostraram correlações negativas entre as dimensões do IPSF e da EVENT conforme o esperado, indicando que quanto maior o suporte familiar, menor a vulnerabilidade ao estresse.

De acordo com a Classificação Brasileira de Ocupações - CBO (Ministério Do Trabalho e Emprego, 2010), os profissionais que exercem a função de porteiro fiscalizam a guarda do patrimônio e exercem a observação de fábricas, armazéns, residências, estacionamentos, edifícios públicos, privados e outros estabelecimentos, percorrendo-os sistematicamente e inspecionando suas dependências, para evitar incêndios, roubos, entrada de pessoas estranhas e outras anormalidades. Além disso, controlam o fluxo de pessoas, identificando, orientando e encaminhando-as para os lugares desejados; recebem hóspedes em hotéis; escoltam pessoas e mercadorias; fazem manutenções simples nos locais de trabalho. Para exercerem a função de porteiro, nas suas várias atribuições, os profissionais precisam apresentar competências pessoais, dentre elas: manter a postura; demonstrar asseio, atenção, educação, trabalho em equipe, paciência, responsabilidade; manter $\mathrm{O}$ autocontrole; organizar-se; administrar seu próprio tempo; estar atualizado; aceitar idéias e aplicar normas de combate a incêndio.

Algumas variáveis presentes no seu contexto de trabalho podem contribuir para o desenvolvimento do estresse, como a exposição a assaltos, a estrutura física das guaritas em que trabalham e carga horária de trabalho. A pressão de moradores, síndicos, donos de estabelecimentos comerciais e supervisores da empresa prestadora de serviços para que o porteiro se responsabilize pela segurança do posto de serviço pode ser também um estressor.

As atividades desenvolvidas pelos porteiros, assim como as variáveis às quais estão expostos, sugerem que estes profissionais podem ser vulneráveis ao estresse. Entretanto, uma maior ou menor vulnerabilidade pode estar relacionada à percepção de suporte familiar de cada porteiro avaliado, que engloba questões relativas às complexas relações que se estabelecem entre os membros de uma família, podendo, ou não, atuar como um agente amortecedor frente aos eventos estressantes. Desta forma, pode-se lançar a hipótese de que quanto menor a percepção de suporte familiar dos porteiros, maior a sua vulnerabilidade ao estresse no trabalho.

O presente estudo teve por objetivo avaliar os níveis de vulnerabilidade ao estresse no trabalho e percepção de suporte familiar em trabalhadores que exercem a função de porteiro em estabelecimentos residenciais e comerciais, bem como investigar possíveis correlações entre estas variáveis. Pretendeuse, também, comparar características da amostra, obtidas por intermédio do questionário de caracterização, com os resultados dos instrumentos utilizados.

\section{Método}

\section{Participantes}

O estudo contou com a participação de 100 funcionários do sexo masculino de uma empresa prestadora de serviços terceirizados exercendo a função de porteiro em postos de serviços comerciais e residenciais. Os participantes trabalham em turnos e escalas diferenciadas, de acordo com a necessidade da empresa e do posto de serviço em que atuam.

O maior número de participantes se concentrou na faixa etária de 20 a 30 anos (31\%), e nas faixas etárias de 31 a 40 anos e 41 a 50 anos com $29 \%$ e $26 \%$, respectivamente. A idade média dos porteiros foi de 37,8 anos, com $\mathrm{DP}=11,3$, sendo que o participante com maior idade tinha 72 anos e o mais jovem 20 anos. Mais da metade dos participantes $(60 \%)$ possuíam o Ensino Médio completo, uma exigência da empresa. Grande parte dos participantes possuía união estável com a parceira $(77 \%)$ e relatou realizar atividades de lazer $(77 \%)$, tais como caminhada, futebol, andar de bicicleta, leitura, passeio, natação, ouvir música, assistir TV e fazer musculação. Os dados também mostraram que $91 \%$ dos participantes relataram não possuir problema de saúde e o restante declarou possuir algum tipo de problema como hérnia, hipertensão, diabete, gastrite, hipoglicemia, colesterol, rinite alérgica e estresse. 
No âmbito profissional, em média, os porteiros trabalhavam na empresa há 4,07 anos $(\mathrm{DP}=4,05)$ e como porteiros, em média, há 5,77 anos $(\mathrm{DP}=5,33)$. Todos os participantes trabalhavam em escalas: $75 \%$ na escala $12 \times 36$ e $25 \%$ na de $5 \times 1$. Observou-se que mais da metade do grupo trabalhava somente no período noturno (57\%), outra parte no diurno $(38 \%)$ e a minoria nos dois períodos $(5 \%)$.

\section{Instrumentos}

Questionário de caracterização - informações dos participantes quanto a: 1) aspectos pessoais como idade, nível de escolaridade, estado civil, número de filhos, tempo semanal direcionado a atividades de lazer e descanso, problemas de saúde, e 2) aspectos profissionais como tempo de empresa e de atuação como porteiro, turno, escala e acidentes de trabalho. Escala de Vulnerabilidade ao Estresse no Trabalho EVENT (Sisto, Baptista, Noronha \& Santos, 2007) que objetiva avaliar a vulnerabilidade da pessoa frente aos estressores no ambiente de trabalho. $O$ instrumento é do tipo Likert de três pontos, no qual os respondentes assinalam 0 para nunca, 1 para às vezes e 2 para frequentemente. As situações apresentadas na escala envolvem a percepção do respondente frente à instituição organizacional, à filosofia da empresa, relacionamentos interpessoais no ambiente de trabalho e de seus sentimentos frente às atividades laborais, tais como motivação e autonomia.

O instrumento foi estruturado em 40 itens e os fatores foram nomeados e divididos em Clima e Funcionamento Organizacional, Pressão no trabalho e Infra-estrutura e Rotina. Pontua-se a EVENT pela soma dos itens assinalados pelo respondente, com pontuação mínima de zero e máxima de 80 , indicando que quanto maior a pontuação obtida, maior a vulnerabilidade ao estresse.

Inventário de Percepção de Suporte Familiar - IPSF (Baptista, 2010) tem por objetivo mensurar o construto de suporte familiar. $O$ instrumento possui 42 itens, divididos em três dimensões, sendo o Fator 1 denominado de Afetivo-Consistente que envolve questões relacionadas à expressão de afetividade entre os membros da família (verbal ou não), interesse, simpatia, acolhimento, consistência de comportamentos e habilidades na resolução de problemas; o Fator 2 Adaptação Familiar que trata dos sentimentos negativos do respondente em relação ao grupo familiar, tais como raiva, exclusão, isolamento e falta de compreensão e o Fator 3 Autonomia avalia as relações de confiança, liberdade e privacidade entre os membros da família. Alguns estudos sobre as evidências de validade de construto e critério para o IPSF são os de Rigotto (2006), Aquino (2007) e Souza
(2007). Ao final da avaliação, quanto maior a pontuação obtida, maior é o nível de suporte familiar percebido pelo respondente.

\section{Procedimento}

Este estudo obedeceu à Resolução 196/96 do Conselho Nacional de Saúde em relação às Diretrizes e Normas Regulamentadoras de Pesquisas envolvendo Seres Humanos, tendo sido aprovado pelo Comitê de Ética em Pesquisa (Processo n ${ }^{\circ}$ 2074/46/01/07).

Em sessão coletiva, fora do horário da jornada de trabalho, os participantes interessados, divididos em grupos que variaram de 3 a 14 pessoas, responderam aos instrumentos na ordem anteriormente apresentada. A duração média da sessão de coleta de dados foi de 45 minutos e para se atingir o total de 100 participantes interessados foram necessárias 13 sessões coletivas. As instruções foram dadas gradualmente de acordo com a ordem de aplicação dos instrumentos e respeitaram as regras de aplicação contidas nos manuais, o que facilitou a compreensão dos participantes e padronizou as instruções para todos os grupos.

Nota-se que alguns instrumentos possuem palavras requintadas, o que poderia ser prejudicial ao resultado final da coleta, pois a falta de compreensão poderia fazer com que os participantes deixassem de responder algum item ou respondessem apenas para seguirem as instruções, mesmo que tais respostas não condissessem com a realidade. Por este motivo, alguns sinônimos foram utilizados para algumas palavras (cefaléia $=$ dor de cabeça; aumento de sudorese $=$ muito suor ou suadeira; insônia $=$ dificuldade de dormir; hipertensão arterial $=$ pressão alta; taquicardia $=$ batedeira no peito; problemas dermatológicos = problemas de pele; sensibilidade emotiva excessiva $=$ estar muito nervoso; hiperventilação = respirar rápido; diminuição da libido $=$ sem vontade de sexo), conforme orientação dos autores dos instrumentos, constante no manual.

\section{Resultados}

$\mathrm{Na}$ análise dos resultados, no Fator Clima e Funcionamento Organizacional da EVENT, a média foi de 9,33 ( $\mathrm{DP}=5,16)$. Dado que o ponto médio considerado pelo instrumento é de 14,15, observa-se que a média obtida e o ponto médio estão bem distantes, indicando que os participantes, neste fator, tenderam a ter pontuações baixas. Com relação à Pressão no Trabalho, a média foi de 10,48 (DP=3,95). Considerando que o ponto médio seria de 12,76 pontos, a média obtida ficou inferior ao ponto médio. Este dado indica, assim como no Fator 1, que os participantes tenderam a apresentar pontuações baixas. 
Quanto a Infra-estrutura e Rotina (Fator 3), a média foi de 6,07 (DP=2,83). Identificando-se um ponto médio de 6,16 , nota-se que a média e o ponto médio ficaram bem próximos, sugerindo que os participantes tenderam a ter pontuações superiores nesse fator. Por fim, no que se refere à medida de vulnerabilidade ao estresse geral, a média foi de 25,9 $(\mathrm{DP}=9,20)$. Sendo o ponto médio dessa escala para este grupo de profissionais de 32,79 pontos, a média e o ponto médio ficaram muito distantes, sugerindo que foram indicados poucos estressores no geral por esse grupo de profissionais, o que pareceu algo esperado, a partir dos dados de cada fator apresentados anteriormente.

Quanto ao suporte familiar, nenhum participante apresentou o escore mínimo nas dimensões, mas houve quem apresentou o escore máximo esperado. A média das respostas na dimensão Afetivo-Consistente foi de 31,62 e $\mathrm{DP}=7,25$, na dimensão Adaptação Familiar foi de 22,08 e DP=2,95, na dimensão Autonomia Familiar foi de 11,82 e $\mathrm{DP}=3,18$ e no Total foi de 65,52 e $\mathrm{DP}=10,66$. Estes dados sugerem que grande parte dos participantes apresentou uma percepção de um bom suporte familiar.

Considerando-se os percentis de pontuação obtidos na validação do instrumento, os porteiros apresentaram, na pontuação total, percentil médio-alto de percepção de suporte familiar. Com relação às outras dimensões, nota-se que os participantes apresentaram percentil médio alto na AfetivoConsistente e médio baixo na Adaptação e Autonomia Familiar.

Os resultados obtidos com a EVENT foram correlacionados com os do IPSF. A pontuação do inventário que mede a percepção de suporte familiar dos participantes do estudo aponta que quanto maior o escore, melhor a percepção de suporte familiar. O instrumento que mede a vulnerabilidade ao estresse no trabalho assinala que quanto menor o escore, menos vulnerável ao estresse o participante se encontra. Com isso, as correlações buscadas nestes instrumentos são negativas, uma vez que a pontuação destes é inversa. Ou seja, quanto menor for o nível de vulnerabilidade ao estresse no trabalho, maior deverá ser o nível do suporte familiar. A Tabela 1 mostra que algumas correlações foram significativas.

Tabela 1 - Coeficiente de Correlação de Pearson entre as Dimensões do IPSF e as Dimensões da EVENT

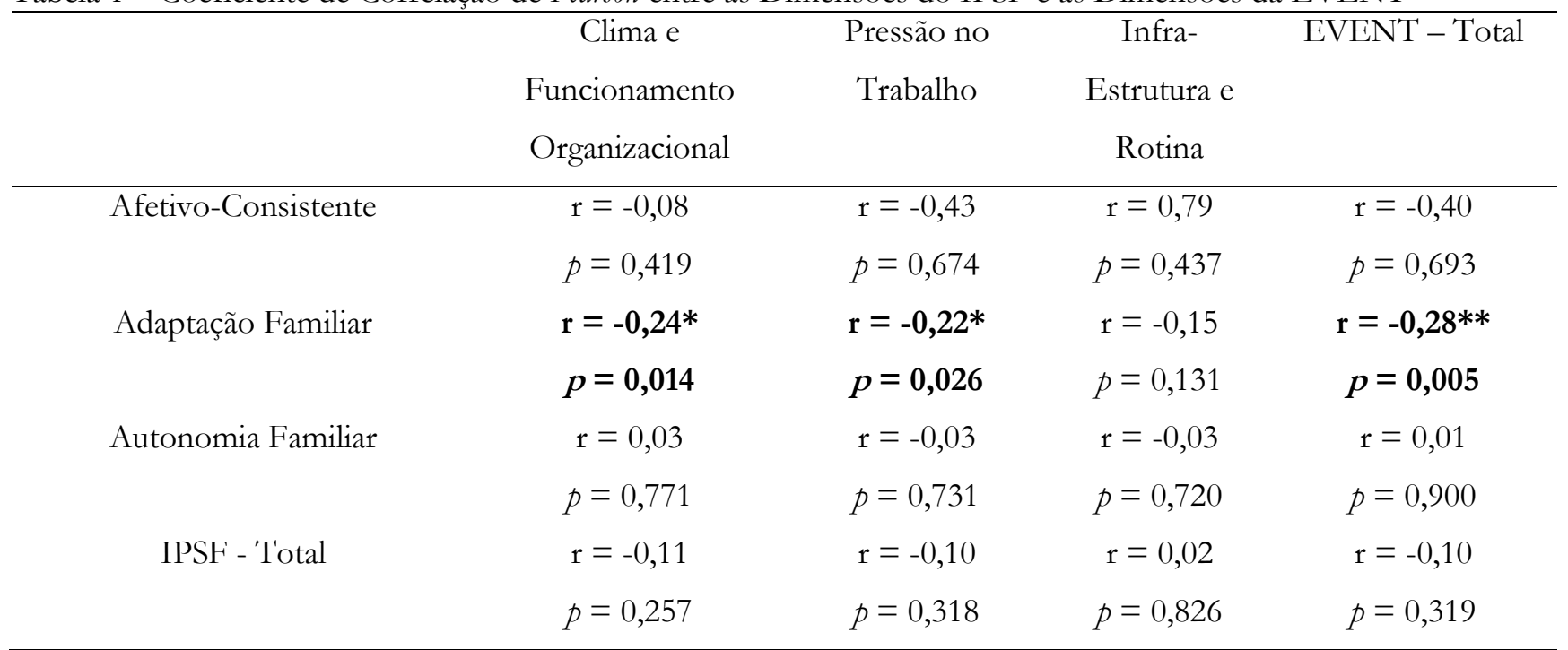

** Correlação é significativa ao nível de 0,01 .

* Correlação é significativa ao nível de 0,05.

A dimensão do IPSF Adaptação Familiar foi a única em que os dados foram significativos estatisticamente, se correlacionada com a EVENT. Adaptação Familiar, portanto, correlacionou-se negativamente com as seguintes dimensões do EVENT: Clima e Funcionamento Organizacional, Pressão no Trabalho e o Total da EVENT. Estes dados indicam que quanto menor a percepção do respondente com relação à sua Adaptação Familiar, maior será sua vulnerabilidade ao estresse nos âmbitos pressão no trabalho, clima, funcionamento organizacional e, principalmente na vulnerabilidade ao estresse total que apresentou uma significância ao nível de $1 \%$

Análises secundárias foram realizadas com o objetivo de comparar características pessoais e profissionais da amostra com os resultados dos instrumentos EVENT e IPSF. Para a realização das 
análises, utilizou-se o teste estatístico não paramétrico U de Mann-Whitney e o Coeficiente de Correlação de Pearson.

As dimensões Afetivo-consistente, Adaptação Familiar e Total do IPSF apresentaram diferença significativa quanto ao estado civil $(\mathrm{U}=615,5 ; \mathrm{p}=0,027$; $\mathrm{U}=559,0 ; \mathrm{p}=0,007$ e $\mathrm{U}=632,5 ; \quad \mathrm{p}=0,038$, respectivamente). O primeiro grupo foi composto de porteiros casados ou com união estável e o segundo grupo, de porteiros divorciados, separados, viúvos e solteiros. Os porteiros casados obtiveram médias superiores aos não-casados em todos os itens apresentados anteriormente, indicando melhor percepção de suporte familiar.

Com relação à variável atividades de lazer houve diferença significativa apenas com o Fator 3 da EVENT (U=646,0 e $\mathrm{p}=0,048)$, indicando maior vulnerabilidade ao estresse na dimensão Infra-estrutura e rotina de trabalho entre os participantes que realizam atividades de lazer. A média deste grupo foi maior $(53,61)$ que a do grupo que relatou não realizar nenhuma atividade de lazer $(40,09)$.

Quanto aos dados profissionais dos porteiros, analisou-se tempo de empresa e de atuação como porteiro, escala, período e acidente de trabalho que foram comparados aos instrumentos utilizados no estudo. Encontrou-se diferença significativa entre IPSF e escala de trabalho; IPSF e tempo de empresa; IPSF e tempo de trabalho como porteiro.

Com relação aos resultados obtidos nos instrumentos quando comparados à variável escala de trabalho, notam-se diferenças significativas entre as médias na dimensão Autonomia Familiar (U=558,0 e $\mathrm{p}=0,002)$ e Total do IPSF $(\mathrm{U}=670,5$ e $\mathrm{p}=0,033)$. Esta análise mostra que os porteiros da escala 12x36 apresentam maior Autonomia Familiar e maior percepção de suporte familiar, se comparados aos que trabalham na escala $5 \times 1$.

Com o objetivo de correlacionar as variáveis tempo de empresa e de atuação como porteiros e os dados obtidos nos instrumentos, utilizou-se $\mathrm{O}$ coeficiente de correlação de Pearson. A dimensão Autonomia Familiar do IPSF apresentou correlação negativa significativa estatisticamente tanto com o tempo de empresa $(\mathrm{p}=0,000)$ como com o tempo de atuação como porteiro $(\mathrm{p}=0,017)$, sugerindo que quanto maior o tempo de atuação na área ou na empresa, menor a autonomia familiar dos participantes. As outras dimensões do IPSF, assim como os dados da EVENT, não apresentaram correlação significativa com a variável tempo de empresa dos participantes.

\section{Discussão}

A caracterização dos participantes do presente estudo pode ser considerada padrão, quando comparada a estudos realizados com profissionais de classe trabalhadora menos favorecida como, por exemplo, motoristas urbanos. Pesquisas desenvolvidas por Battiston, Cruz e Hoffmann (2006); Zanelato (2008) também obtiveram dados semelhantes, ou seja, predominância do sexo masculino, idade média de quarenta anos, casados e de baixa escolaridade (no máximo ensino fundamental completo). Apenas os dados referentes à escolaridade foram diferenciados, visto que no presente estudo mais da metade dos porteiros possuía ensino médio completo, uma exigência da empresa.

Os resultados obtidos com a Escala de Vulnerabilidade ao Estresse no Trabalho - EVENT, no geral, indicaram poucos estressores para o grupo estudado. Os dois primeiros fatores avaliados pela EVENT também apresentaram baixos escores, indicando que os porteiros possuem baixa vulnerabilidade ao estresse no que tange ao clima e funcionamento organizacional e pressão no trabalho. $\mathrm{O}$ Fator 3 da escala (infra-estrutura e rotina de trabalho) foi o único a apresentar vulnerabilidade média-alta. Dado semelhante pode ser encontrado no estudo de Bachion e colaboradores (2005) que, ao estudar professores universitários, encontrou a maioria com baixa vulnerabilidade ao estresse. Os resultados encontrados podem dever-se à associação positiva que a empresa exerce sobre a sua atuação, englobando uma cultura adequada e líderes não autoritários ou ao fato de trabalharem em uma cidade que oferece menos riscos e mais segurança, se comparada a cidades metropolitanas.

Quanto ao suporte familiar percebido pelos porteiros e identificado pelo IPSF, houve um percentil médio-alto no total geral avaliado. A dimensão AfetivoConsistente do IPSF também apresentou percentil médio-alto e as outras duas dimensões, Adaptação e Autonomia Familiar mostraram percentil médio-baixo. Os resultados apontam, portanto, para a percepção de um bom suporte familiar entre os participantes do estudo, no geral, com a constatação de percepção de suporte familiar médio-alto.

A primeira análise demonstrou correlação negativa entre a dimensão Adaptação Familiar do IPSF e as dimensões Clima e Funcionamento Organizacional, Pressão no Trabalho e o Total da EVENT, indicando que quanto mais independência e autonomia os porteiros exercitarem na família, menos vulnerabilidade ao estresse no trabalho eles terão. Os dados corroboram os encontrados por Aquino (2007) e estão de acordo com Baptista (2005) por ressaltar o papel do suporte familiar como amortecedor dos 
efeitos estressantes. Entretanto, as outras dimensões do IPSF não apresentaram correlações significativas com a EVENT, contrariando a hipótese inicial e sugerindo que dificilmente este dado seria encontrado pelo fato da maioria dos porteiros ter apresentado a percepção de um bom suporte familiar e uma baixa vulnerabilidade ao estresse.

Sobre as análises secundárias, os resultados encontrados entre as características pessoais dos porteiros e os instrumentos demonstraram haver diferença significativa entre estado civil e IPSF; e atividades de lazer e a dimensão Infra-estrutura e rotina da EVENT. As variáveis idade, escolaridade, número de filhos e horas destinadas ao lazer e descanso, por sua vez, não apresentaram diferenças estatisticamente significantes. Estes dados são contrários à literatura que apresenta estudos que mostram diferenças significativas entre outras variáveis, dentre elas: percepção de suporte familiar e gênero (Dias, 2008; Rigotto, 2006; Santana, 2008); vulnerabilidade ao estresse no trabalho e sexo (Miguel, 2006); percepção de suporte familiar e sexo, raça e configuração familiar (Souza, 2007).

Entretanto, há resultados que corroboram a literatura, como a diferença encontrada por Aquino (2007) entre estado civil e percepção de suporte familiar, que verificou que em relação à dimensão Afetivo-Consistente e ao Total de suporte familiar, a média do grupo de casados foi maior, indicando que estes possuíam mais suporte afetivo-consistente do que os não casados (solteiros, viúvos e divorciados). Estes dados sugerem que o suporte oferecido pelo cônjuge pode fazer com que o outro tenha a percepção de um bom suporte familiar e a possibilidade de possuir uma maior rede de suporte em diferentes situações. Algumas ausências de diferenças significativas deste estudo também corroboram a literatura, tal como idade, escolaridade e vulnerabilidade ao estresse no trabalho (Miguel \& Noronha, 2007; Miguel, 2006).

As características profissionais dos porteiros indicaram diferença significativa entre as variáveis tempo de empresa e de atuação como porteiro, escala de trabalho e o instrumento IPSF. A variável período de trabalho não apresentou dado significativo com nenhum instrumento. Os dados da EVENT, por sua vez, também não apresentaram significância estatística com nenhuma variável profissional.

Com relação ao IPSF, não foram encontrados estudos que comparassem este instrumento com as variáveis de caracterização tempo de atuação e de empresa e escala de trabalho, demonstrando que outras pesquisas se fazem necessárias para confirmar ou refutar este dado. Entretanto, o estudo de Aquino (2007) encontrou diferenças significativas entre o IPSF e outros dados sociodemográficos, tais como carga horária e tempo gasto no trabalho. Santana (2008) também encontrou diferença significativa entre percepção de suporte familiar e atividades de lazer, confirmando a hipótese de que havia diferença entre as pontuações do IPSF e suas dimensões, de acordo com o tempo gasto com atividades de lazer com a família, ou seja, de que havia diferença na percepção de suporte familiar dependendo de quanto tempo o participante passava em atividade de lazer com a família. Isto mostra que os porteiros apresentam resultados diferenciados dos encontrados em outros estudos por fazerem parte de uma classe de trabalhadores pouco estudada e que, possivelmente, está exposta a condições diferenciadas no âmbito familiar, pessoal e profissional.

A variável período de trabalho não se correlacionou significativamente com nenhum instrumento, indicando ausência de diferenças entre os porteiros que trabalham no período noturno e diurno. Este dado contraria a discussão de Dela Coleta (2007) ao apontar que o trabalho em diferentes turnos é um fator psicossocial desfavorável ao bem estar do trabalhador, podendo acarretar em prejuízos devido a não adaptação do sujeito a diferentes horários de trabalho.

A variável escala de trabalho apresentou diferenças significativas com a dimensão Autonomia Familiar e Total do IPSF. Uma hipótese para a média dos participantes na escala $12 \times 36$ ter sido maior que a média na $5 \times 1$ é o fato dos porteiros que atuam na escala $12 \times 36$ possuírem um maior tempo disponível para o contato familiar por terem a possibilidade de descansar 36 horas e trabalhar 12 horas, ao contrário do outro grupo que precisa trabalhar cinco dias consecutivos para folgar apenas um.

Nenhum dado de caracterização da amostra apresentou resultado estatisticamente significante com a EVENT, exceto com a variável atividades de lazer, que apresentou um dado no limite da significância, sugerindo que a vulnerabilidade ao estresse no trabalho não tem associação com as características pessoais e profissionais dos porteiros. Estes resultados contrariam os obtidos por Aquino (2007) que encontrou diferença significativa entre a EVENT e algumas variáveis de caracterização: quantidade de tarefas e compatibilidade com a função dos participantes, cursos por áreas, tipo de trabalho exercido, tempo gasto no trabalho e carga horária. Outros estudos, entretanto, também não encontraram diferenças significativas entre EVENT, idade e escolaridade (Miguel \& Noronha, 2007; Miguel, 2006) e entre EVENT e nível econômico (Aquino, 2007), sugerindo que a vulnerabilidade ao estresse pode 
estar presente no sujeito independentemente destas variáveis.

Portanto, parece que a relação entre a maioria dos dados sociodemográficos com a vulnerabilidade ao estresse no trabalho e a percepção de suporte familiar ainda não está bem estabelecida na literatura nacional, necessitando-se de mais estudos com amostras maiores e diferenciadas. Além disso, os resultados não corroboram muitos outros estudos, provavelmente devido às características próprias da população estudada.

\section{Considerações Finais}

Os resultados do presente estudo indicaram a existência de um grupo profissional com baixa vulnerabilidade ao estresse no trabalho e percepção, no geral, de um bom suporte familiar. Dados como estes não são encontrados facilmente na literatura, visto a maioria das profissões estudadas apresentar resultados negativos no que tange a estas variáveis. Os resultados encontrados, contrariando as hipóteses iniciais, sugerem que, ao contrário de outras profissões estudadas, as condições de trabalho na portaria sejam mais favoráveis a um bom estado físico e psicológico dos porteiros, estruturas físicas adequadas, pouca pressão na rotina diária e poucos conflitos entre a equipe de trabalho.

Sugere-se, também, que a empresa prestadora de serviços, da qual os porteiros participantes são funcionários, possa ser o diferencial devido aos procedimentos adequados adotados junto a seus funcionários, indicando uma cultura onde os valores morais e éticos são preservados, dentre outras condições já comentadas. Neste sentido, outros estudos comparando grupos de porteiros de outras empresas da mesma cidade ou de cidades diferentes deveriam ser conduzidos a fim de explicar melhor os dados, comprovando ou refutando tal hipótese.

\section{Referências}

Aquino, R. R. (2007). Inventário de Percepscão de Suporte Familiar (IPSF) e Escala de Vulnerabilidade ao Estresse no Trabalho (EVENT): Evidência de Validade. (Dissertação de Mestrado em Psicologia). Itatiba: Universidade São Francisco.

Bachion, M. M., Abreu, L. O., Godoy, L. F. \& Costa, E. C. (2005). Vulnerabilidade ao estresse entre professores de uma universidade pública. Revista de Enfermagem - UERJ, 13(1), 32-37.

Baptista, M. N. (2010). Inventário de Percepção de Suporte Familiar- IPSF. Manual Técnico. São Paulo: Vetor.
Baptista, M. N. (2007). Inventário de Percepção de Suporte Familiar (IPSF): estudo componencial em duas configurações. Psicologia: Ciência e Profissão, 27(3), 496-509.

Baptista, M. N. (2005). Desenvolvimento do Inventário de Percepção de Suporte Familiar (IPSF): estudos psicométricos preliminares. Psico-USF, 10(1), 1119.

Baptista, M. N., Baptista, A. S. D. \& Dias, R. R. (2001). Estrutura e suporte familiar como fatores de risco na depressão de adolescentes. Psicologia, ciência e profissão, 21(2), 52-61.

Baptista, M. N., Baptista, A. S. D. \& Torres, E. C. R. (2006). Associação entre suporte social, depressão e ansiedade em gestantes. PSIC - Revista de Psicologia da Vetor Editora, 7(1), 39-48.

Baptista, M. N. \& Oliveira, A. A. (2004). Sintomatologia de depressão e suporte familiar em adolescentes: um estudo de correlação. Revista Brasileira de Crescimento e Desenvolvimento Humano, 14(3), 58-67.

Battiston, M., Cruz, R. M. \& Hoffmann, M. H. (2006). Condições de trabalho e saúde de motoristas de transporte coletivo urbano. Estudos de Psicologia, 11(3), 333-343.

Calais, S. L. (2004). Stress pós-traumático: intervenção em vítimas secundárias. Em M. E. N. Lipp (Org.). O stress no Brasil: pesquisas avançadas (pp. 121-130). Campinas, SP: Papirus.

Carvalho, I. M. M. \& Almeida, P. H. (2003). Família e Proteção Social. São Paulo em Perspectiva, 17(2), 109122.

Covolan, M. A. (2001). Stress ocupacional do psicólogo clínico: Seus sintomas, suas fontes e as estratégias utilizadas para controlá-lo. Em M. E. N. Lipp (Org.). Pesquisas sobre stress no Brasil (pp. 225-240). Campinas, SP: Papirus.

Dela Coleta, A. S. M. (2007). Estresse e suporte social em profissionais do setor de segurança pessoal e patrimonial. (Dissertação de Mestrado em Psicologia Aplicada). Uberlândia: Universidade Federal de Uberlândia.

Dias, C. C. (2008). Evidências de validade da escala de depressão (EDEP) em uma amostra no contexto hospitalar. (Dissertação de Mestrado em Psicologia). Itatiba: Universidade São Francisco.

Ferachin, L. K., Ranzi, P., Garib, S. M. \& Lopes, S. A. (2004). "Ser melhor": Conhecendo o stress, vivendo com qualidade de vida, um trabalho com policiais militares. Em M. Z. Brandão (Org.). Sobre Comportamento e Cognição: entendendo a psicologia comportamental e cognitiva aos contextos da saúde, das organizaçôes, das relaçoes pais e filhos e das escolas (pp. 71-75). Santo André, SP: ESETec Ed. Associados. 
Limongi-França, A. C. L. \& Rodrigues, A. L. (2005). Stress e trabalho: guia prático com abordagem psicossomática. 5 ed. São Paulo: Atlas.

Lipp, M. E. N. (2000). Inventário de Sintomas de Stress para Adultos de Lipp (ISSL). São Paulo: Casa do Psicólogo.

Lipp, M. E. N. (1999). O stress está dentro de você. São Paulo: Contexto.

Lipp, M. E. N. \& Tanganelli, M. S. (2002). Stress e qualidade de vida em magistrados da Justiça do Trabalho: Diferenças entre homens e mulheres. Psicologia: Reflexão e Crítica. Rio Grande do Sul, 15(3), 537-548.

Maciel, S. V. (2001). Atleta juvenil feminina: correlação entre características psicológicas, stress e lesões osteomusculares. Em M. E. N. Lipp (Org.). Pesquisas sobre stress no Brasil (pp. 211-224). Campinas, SP: Papirus.

Miguel, F. K. (2006). Estresse e Inteligência Emocional: evidências de validade. (Dissertação de Mestrado em Psicologia). Itatiba: Universidade São Francisco.

Miguel, F. K.; Noronha, A. P. P. (2007). Estudo dos Parâmetros Psicométricos da Escala de Vulnerabilidade ao Estresse no Trabalho. Evaluar, 7, 1-18.

Ministério do Trabalho e Emprego. Classificação brasileira de ocupaçooes. Brasília. Disponível em: $<$ http://www.mtecbo.gov.br/busca/descricao.asp ?codigo $=5174>$. Acesso em: 20 setembro 2010.

Mota, E. L. A., Franco, A. L. S. \& Motta, M. C. (1999). Migração, estresse e fatores psicossociais na determinação da saúde da criança. Psicologia: Reflexão e Crítica, 12(1), 119-132.

Oliveira, J. B. (2004). Stress. diferenças de gênero em amostra de juízes e servidores públicos. Em M. E. N. Lipp (Org.). O stress no Brasil: pesquisas avançadas (pp. 187-196). Campinas, SP: Papirus.
Paschoal, T. \& Tamayo, A. (2004). Validação da Escala de Estresse no Trabalho. Estudos de Psicologia, 9(1), 45-52.

Paschoal, T. \& Tamayo, A. (2005). Impacto dos valores laborais e da interferência família-trabalho no estresse ocupacional. Psicologia: Teoria e Pesquisa, 21(2), 173-180.

Poletto, R. C. \& Koller, S. H. (2002). Rede de apoio social e afetivo de crianças em situação de pobreza. Psico - Porto Alegre, 33(1), 151-176.

Rigotto, D. M. (2006). Evidências de Validade entre suporte familiar, suporte social e autoconceito. (Dissertação de Mestrado em Psicologia). Itatiba: Universidade São Francisco.

Romano, A. S. P. F. (2001). Stress na polícia militar: proposta de um curso de controle do stress. Em M. E. N. Lipp (Org.). Pesquisas sobre stress no Brasil (pp. 195-210). Campinas, SP: Papirus.

Santana, P. R. (2008). Suporte Familiar, Estilos Parentais e Sintomatologia Depressiva: Um Estudo Correlacional. (Dissertação de Mestrado em Psicologia). Itatiba: Universidade São Francisco.

Souza, M. S. (2007). Suporte Familiar e Saúde Mental: evidência de validade baseada na relação entre variáveis. (Dissertação de Mestrado em Psicologia). Itatiba: Universidade São Francisco.

Sisto, F. F., Baptista, M. N., Noronha, A. P. \& Santos, A. A. A. (2007). Escala de Vulnerabilidade ao Estresse no Trabalho - EVENT. São Paulo: Vetor.

Zanelato, L. S. (2008). Manejo de stress, coping e resiliência em motoristas de onibus urbano. (Dissertação de Mestrado em Psicologia do Desenvolvimento e Aprendizagem). Bauru: Universidade Estadual Paulista.

Recebido em novembro de 2010 Reformulado em janeiro de 2011 Aprovado em março de 2011

Sobre as autoras:

Cristiane Oliveira Alves Telles Nunes é graduada e mestre em Psicologia pela Universidade Estadual Paulista Júlio de Mesquita Filho (UNESP-Bauru), docente do Centro Universitário de Votuporanga (UNIFEV) e doutoranda em Psicologia pela Universidade Federal de São Carlos (UFSCar).

Sandra Leal Calais é psicóloga, doutora em Psicologia pela Pontifícia Universidade Católica de Campinas, Professora-Assistente Doutor da Universidade Estadual Paulista Júlio de Mesquita Filho - Unesp, Campus Bauru, onde está vinculada ao Departamento de Psicologia, ao Centro de Psicologia Aplicada (CPA) e ao Programa de Pós-Graduação em Psicologia do Desenvolvimento e Aprendizagem da Faculdade de Ciências. 